\title{
Optical Studies in ZnS:Ce Nanocrystallite
}

\author{
GEORGE VARUGHESE $^{1 *}$ and K. T. USHA ${ }^{2}$ \\ ${ }^{1}$ Department of Physics, Catholicate College, Pathanamthitta, Kerala-689645, India \\ ${ }^{2}$ Department of Chemistry, St. Cyrils College, Adoor, Kerala \\ gvushakoppara@yahoo.co.in
}

Received 2 August 2014 / Accepted 5 September 2014

\begin{abstract}
Cerium doped $\mathrm{ZnS}$ nanoparticles were prepared by chemical route. The particle size was determined from the X-ray line broadening. Samples were characterized by XRD, FTIR and UV and SEM. The composition was verified by EDAX spectrum. The size of the particles increased as the annealing temperature was increased. The crystallite size varied from $21.7 \mathrm{~nm}$ to $25 \mathrm{~nm}$ as the calcination temperature increased. Band gap values $\mathrm{Ce}^{3+}$ doped $\mathrm{ZnS}$ were determined to $3.86 \mathrm{eV}$ from the optical transmission studies of the as-prepared samples and exhibited a redshift towards $320 \mathrm{~nm}$ in comparison with undoped $\mathrm{ZnS}$ nanoparticles. The redshift of the absorption edge to the longer wavelength side has been attributed to the strong exchange interaction between the $\mathrm{d}$ electron of $\mathrm{Ce}$ and $\mathrm{s}$ and $\mathrm{p}$ electron of the $\mathrm{ZnS}$ host band.
\end{abstract}

Keywords: Semiconductor, Nanomaterial, Doping, Ultrasonic velocity

\section{Introduction}

It is well-known that the optical and electronic properties change dramatically due to quantum confinement of the charge carriers within the particle. $\mathrm{ZnS}$, which is an important wide band gap semiconductor, has potential applications in electronics and optoelectronics including light emitting diodes, efficient phosphors in flat-panel displays and photo voltaic devices because of its wide direct band gap $3.77 \mathrm{eV}^{1,2}$. One Dimensional $\mathrm{ZnS}$ nano particle have been attracting growing attention because they possess unique properties compared to bulk crystal due to quantum confinement and surface effect ${ }^{3}$. Doped semiconductor nanoparticle $\mathrm{ZnS}: \mathrm{Ce}$ is used as phosphors and also in thin film electroluminescent devices ${ }^{4}$. Recently $\mathrm{ZnS}$ nanostructures have shown, a great promise and functional structure nanobuilding blocks in nanoelectronics, nano-optoelectronics and nanolaser ${ }^{5,6} . \mathrm{ZnS}$ has been used in many military applications requiring mechanical resistance to hostile environment, missile domes and external windows of military aircraft. Since the mechanical properties are crucial for designing such devices there is an increasing interest in the elasticity of nanostructures. Band gap energy decreases on doping with $\mathrm{Ce}$. The cerium ion doping will change carrier concentration in the $\mathrm{ZnS}$ nano particles ${ }^{7,8}$. The dopant sites, defects induced by doping have strong impact on the Structural, Mechanical and Optical properties of $\mathrm{ZnS}^{9}$. Since $\mathrm{CeO}_{2}$ has a band gap of $\sim 3 \mathrm{eV}$ and the fascinating properties that are similar to shows that 
of $\mathrm{ZnS}$, the mixed of both materials have been investigated for the probing of highly efficient Photo catalysis ${ }^{10}$. The dopant sites defects induced by the doping have strong impact on the structural and optical properties of Ce doped $\mathrm{ZnS}^{11}$. In this paper we report the study on the effect of Ce ion as a dopant in $\mathrm{ZnS}$ nanoparticle. The effect of Ce doping on the optical properties was evaluated.

\section{Experimental}

Nanoparticles of Ce doped ZnS were prepared by chemical coprecipitation method ${ }^{12}$. All the chemicals were of AR grade and were used without further purification. For the synthesis of $\mathrm{Ce}^{3+}$ doped $\mathrm{ZnS}, 3 \mathrm{~g}(0.35 \mathrm{M})$ of zinc acetate in $40 \mathrm{~mL}$ of deionized water-ethanol matrix (equal volumes) and $1.5 \mathrm{~g}(0.39 \mathrm{M})$ of $\mathrm{Ce}(\mathrm{II})$ chloride hepta hydrate in $10 \mathrm{~mL}$ of water were mixed drop by drop. The entire mixture was stirred magnetically at $80{ }^{\circ} \mathrm{C}$ until a homogeneous solution was stirred. The $3 \mathrm{~g}(1 \mathrm{M})$ of thiourea in $40 \mathrm{~mL}$ of deionized water ethanol matrix was added to the above mixture. The entire solution was stirred magnetically until a white precipitate was formed. The obtained dispersion was purified by dialysis against deionized water and acetone several times to remove impurities. Few drops of Tri Ethyle Amine (TEA) was added to the solution to reduce the agglomeration of particles to remove the impurities, including traces of TEA and the original reactants, if any. The wet precipitate was dried in the controlled temperature furnace at $120{ }^{\circ} \mathrm{C}$ for $2 \mathrm{~h}$ and thoroughly pulverized.

X-ray diffraction (XRD) and Fourier Transform Infrared (FTIR) analyses of these samples were done for their characterization. XRD patterns were recorded on a RigaKuC/max-2500 diffractometer using graphite filtered $\mathrm{CuK} \alpha$ radiation $(\lambda=1.54056 \AA)$ at $40 \mathrm{kV}$ and $100 \mathrm{~mA}$ with a scanning rate of $8^{\circ}$ per min from $2 \mu=5^{\circ}$ to $80^{\circ}$. The FTIR spectra were recorded in an FTIR spectrometer (Nicolet Magna-750) in the range of $500 \mathrm{~cm}^{-1}$ to $4000 \mathrm{~cm}^{-1}$. The percentage of doped impurity is measured from EDAX spectrum. The Energy Dispersive Analysis of $\mathrm{X}$-rays (EDAX) was done on the sample to ascertain the composition. Scanning Electron Microscopy (SEM) uses electrons to form an image. It is used to show sub-surface defects. In the present study, the UV measurements were done with UV spectrometer JASCO V-550 in the wavelength range of $200 \mathrm{~nm}$ to $850 \mathrm{~nm}$. The UV absorption peak of the prepared nanocrystal was measured and computed the optical band with energy.

\section{Results and Discussions}

\section{XRD analysis}

XRD patterns, of the samples at two different temperatures were carried out. As prepared sample was calcined at 150 and $180{ }^{\circ} \mathrm{C}$ for $5 \mathrm{~h}$ in air atmosphere to ascertain the formation of different nanocrystalline phases. The patterns are compared with Joint Committee for Powder Diffraction Scan (JCPDS) File No. 01-089-7385. Particle Size, are evaluated and tabulated in Table 1, by the formula ${ }^{13,14}$, Debye- Scherrer's formula

$$
\mathrm{L}=\mathrm{K} \lambda / \beta \cos \theta
$$

$\mathrm{K}=0.89, \lambda$ the $\mathrm{X}$-ray wavelength $=0.154095 \mathrm{Nm}, \beta$ the full wavelength at half maximum and $\theta$ the half diffraction angle. Particles annealed at temperatures $150{ }^{\circ} \mathrm{C}$ and 180 ${ }^{0} \mathrm{C}$ have grain sizes $21.7 \mathrm{~nm}$ and $25 \mathrm{~nm}$. A continuous increase in the particle size with temperature was observed. According to Ostwald ripening ${ }^{14}$ the increase in the particle size is due to the merging of the smaller particles into larger and is a result of potential energy difference between small and large particles and can occur through solid state diffusion. From the $\mathrm{X}$ - ray diffraction analysis the crystallite size of $\mathrm{Ce}^{3+}$ doped $\mathrm{ZnS}$ and undoped $\mathrm{ZnS}$ were estimated around $22.7 \mathrm{~nm}$ and $5.3 \mathrm{~nm}$ respectively. Such an increase in particle size is owing to the broadening of diffraction peaks decreased on cerium doping. 
Table 1. Variation of grain size of $\mathrm{ZnS}$ :Ce with temperature

\begin{tabular}{cccccc}
\hline $\begin{array}{c}\text { Temperature } \\
{ }^{\circ} \mathrm{C}\end{array}$ & FWHM & $\beta \times 10^{3}$ & $2 \theta$ & $\theta$ & $\begin{array}{c}\text { Particle } \\
\text { size }(\mathrm{L}) \mathrm{nm}\end{array}$ \\
\hline 150 & 0.364 & 6.34 & 12.875 & 6.4375 & 21.7 \\
180 & 0.312 & 5.44 & 13.352 & 6.676 & 25 \\
\hline
\end{tabular}

\section{SEM Image of $\mathrm{ZnS}: \mathrm{Ce}$}

The morphology, analysis of die/package cracks and fracture surfaces, bond failures, physical defects on the die or package surfaces and dimension of the sample can be studied using SEM. The scanning electron micrographs of $\mathrm{ZnS}$ nanomaterials synthesized under aqueous medium. The orientation growth of $\mathrm{ZnS}$ crystal in water is higher ${ }^{15}$. Spherical shaped morphology is observed in the micrograph of $\mathrm{ZnS}: \mathrm{Ce}$ (Figure 1). The SEM pictures exhibit spherical morphology with self aligned prismatic nanoparticles. The morphology of $\mathrm{ZnS}$ nanopowder as revealed by FESEM showed nanoparticles of size 15-100 Nm.

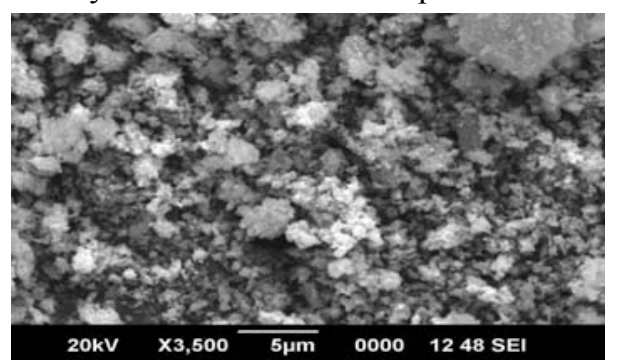

Figure 1. SEM images of the prepared $\mathrm{ZnS}$ :Ce nanocrystals

\section{$E D A X(E D S)$ spectrum of $\mathrm{ZnS}: \mathrm{Ce}$}

EDS (EDAX) is a technique used for identifying the elemental composition of the specimen. In EDS spectrum, (Figure 2) each of the peaks is unique to an atom, and therefore corresponds to a single element. The higher a peak in a spectrum, the more concentrated the element is in the spectrum. An EDS spectrum plot not only identifies the element corresponding to each of its peaks, but the type of X-ray to which it corresponds as well of our sample. About $97.22 \%$ of $\mathrm{Zn}^{2+}$ ion and about $2.28 \%$ Ce ion by mass are present in the sample.

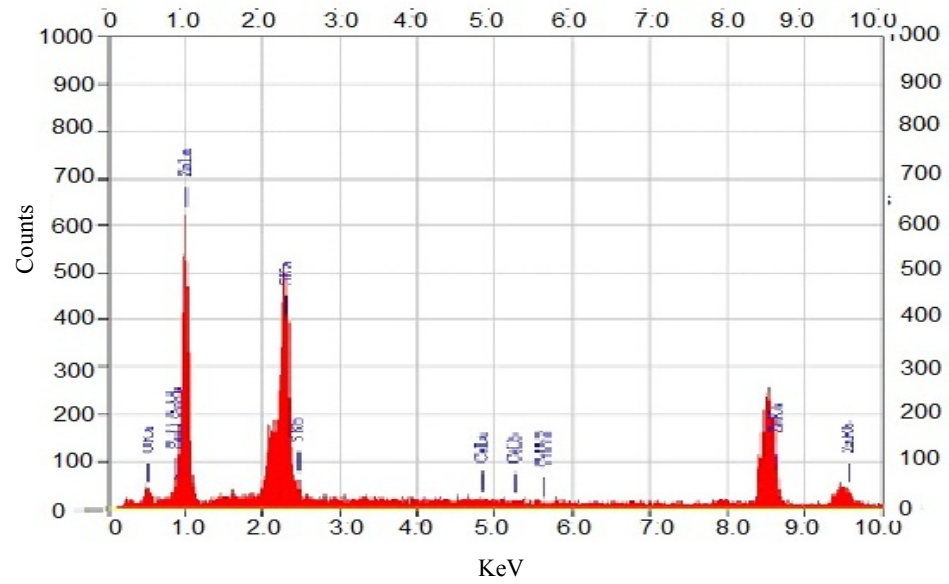

Figure 2. EDAX spectrum of $\mathrm{ZnS}$ :Ce nanoparticle 


\section{Optical studies}

\section{FTIR spectrum of $\mathrm{ZnS}: \mathrm{Ce}$}

The graph depicted in Figure 3 represents the percentage variation of transmittance with wavenumber $\left(\mathrm{cm}^{-1}\right)$. For $\mathrm{ZnS}: \mathrm{Ce}$ annealed at $350{ }^{\circ} \mathrm{C}$ the absorption peaks were observed at $3396.15 \mathrm{~cm}^{-1}, 1625.88 \mathrm{~cm}^{-1}, 1545.39 . \mathrm{cm}^{-1}, 1384.10 \mathrm{~cm}^{-1}, 1019.87 \mathrm{~cm}^{-1}, 829.06 \mathrm{~cm}^{-1}, 675.89 \mathrm{~cm}^{-1}$ and $608.40 \mathrm{~cm}^{-1}$. The FTIR measurements were undertaken in order to confirm the formation of crystalline $\mathrm{ZnS}$ nanoparticle and identify any adsorbed species on to the crystal surface. Bands at $418.56 \mathrm{~cm}^{-1}$ is assigned to the stretching vibrations of $\mathrm{Zn}-\mathrm{S}^{16}$. The stretching frequency of bulk $\mathrm{ZnS}$ is $483.72 \mathrm{~cm}^{-1}$. Three intense bands are centered at $1384.08 \mathrm{~cm}^{-1}$, $1023.37 \mathrm{~cm}^{-1}$ and $1550.06 \mathrm{~cm}^{-1}$ and are attributed to the stretching vibrations of $\mathrm{C}=\mathrm{O}, \mathrm{C}=\mathrm{C}$ and $\mathrm{C}$-H groups in acetate species, which suggest it presents as absorbed species in the surface of nanoparticles. The broad absorption peak centered at $3389.47 \mathrm{~cm}^{-1}$ and $2922.47 \mathrm{~cm}^{-1}$ corresponds to $\mathrm{O}-\mathrm{H}$ stretching indicating the existence of water in the surface of nanoparticles $^{16,17}$.

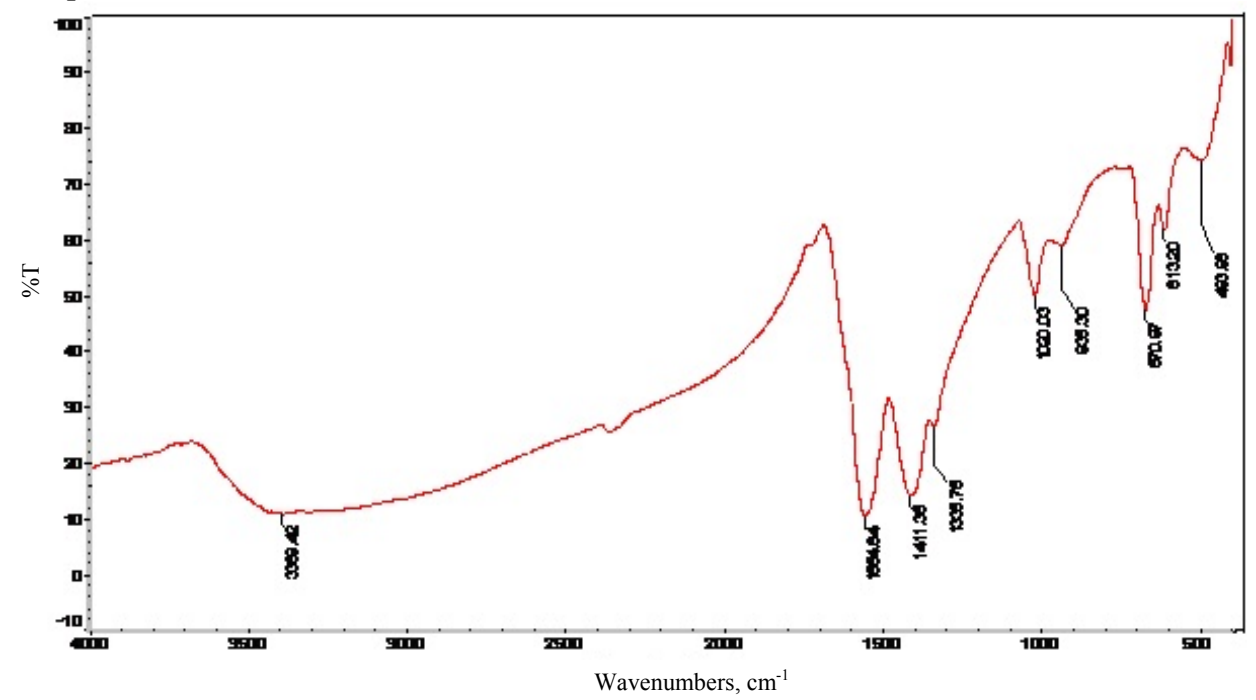

Figure 3. FTIR Spectrum of ZnS:Ce nanoparticle

\section{UV-Vis absorption of $\mathrm{ZnS}: \mathrm{Ce}$}

From Figure 4, it can be seen that the strongest absorption edge of the prepared sample appears at around $320 \mathrm{~nm}$, which is fairly blue-shifted from the absorption edge of the bulk $\mathrm{ZnS}(345 \mathrm{~nm})$ and red shifted with respect to undoped $\mathrm{ZnS}$ nanoparticle $(299 \mathrm{~nm})$. Semiconductor crystallites in the diameter range of a few nanometers show a three dimensional quantum size effect in their electronic structure ${ }^{18}$. The relation between absorption coefficient $(\alpha)$ and incident photon energy $(h v)$ can be written as ${ }^{19}$.

$$
\alpha=A(h v-E \mathrm{~g})^{n}=h v
$$

Where $A$ is a constant and $E_{\mathrm{g}}$ is the band gap of the material. Exponent $n$ depends on the type of the transition; $n$ has value $1 / 2$, as it corresponding to direct transitions. It was noticed that the optical band gap value of undoped $\mathrm{ZnS}$ nanoparticle, $4.13 \mathrm{eV}$, which is higher than the bulk value of $\mathrm{ZnS}, 3.68 \mathrm{eV}^{17}$. From the figure band gap of $\mathrm{Ce}^{3+}$ doped $\mathrm{ZnS} 3.86 \mathrm{eV}$ which can be attributed to the quantum confinement of $\mathrm{ZnS}$ nanoparticle. 


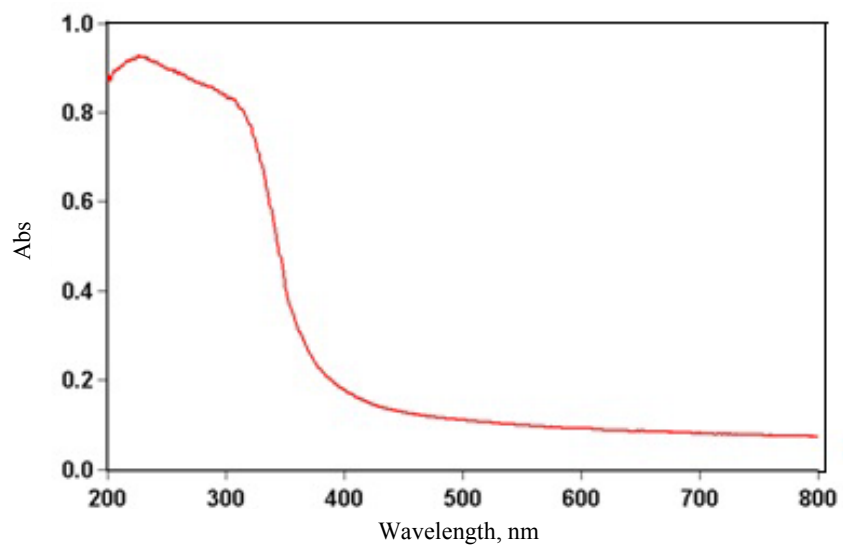

Figure 4. UV- VIS absorption spectrum

The blue shift can be explained on the basis of Burstein-Moss effect. According to which the increase in carrier concentration due to doping of $\mathrm{Ce}^{3+}$ results in a shift of the Fermi level and block some of the lowest states, thereby causing widening of the band resulting in the blue shift of the absorption tail. The change in band gap along with exciton features can be used as a measure of particle size and size distribution ${ }^{20}$. The absorption edge of the $\mathrm{Ce}^{3+}$ doped $\mathrm{ZnS}$ has been shifted to longer wavelength side, due to the co-valent bonding of $\mathrm{Ce}$ with $\mathrm{ZnS}$. As the absorption edge of $\mathrm{Ce}^{3+}$ doped $\mathrm{ZnS}$ is shifted to longer wavelength, the band gap might be decreased compared to undoped $\mathrm{ZnS}$ due to the introduction of new energy in the surface band gap of $\mathrm{ZnS}$ nanoparticle ${ }^{18}$. The shift of the absorption edge to the longer wavelength side has been attributed to the strong exchange interaction between the $d$ electron of Ce and $s$ and $p$ electron of the $\mathrm{ZnS}$ host band ${ }^{21}$. The $\mathrm{Ce}^{3+}$ doped $\mathrm{ZnS}$ is highly effective and can significantly enhance the photo catalytic degradation ${ }^{19}$.

\section{Conclusion}

The structure, optical properties, band gap and composition of Ce doped ZnS nanoparticles were determined by XRD, UV-Vis and EDAX spectra analyses. The strongest UV absorption edge appears at around $380 \mathrm{~nm}$, which is fairly blue-shifted from the absorption edge of the bulk $(345 \mathrm{~nm})$. The XRD results indicated that the particle size of nano $\mathrm{ZnS}: \mathrm{Ce}$ is much small as compared to that of pure nano $\mathrm{ZnS}$ and increases with the cerium loading. Such an increase in particle size is owing to the broadening of diffraction peaks decreased on cerium doping. From the XRD results, it is clear that as temperature increases, particle size also increases. The change in particle size cause large variation in the physical properties since $1 \mathrm{~nm}$ size change may introduce a considerable change in the number of surface atoms with lower coordination and broken exchange bonds. Band gap values Ce doped $\mathrm{ZnS}$ were determined to $3.86 \mathrm{eV}$ from the optical transmission studies of the as-prepared samples. The redshift of the absorption edge to the longer wavelength side has been attributed to the strong exchange interaction between the $d$ electron of Ce and $s$ and $p$ electron of the ZnS host band. The UV Absorption spectra show a shift towards $320 \mathrm{~nm}$. The Ce doped $\mathrm{ZnS}$ is highly effective and can significantly enhance the photo catalytic degradation.

\section{Acknowledgement}

We express our sincere gratitude to the SPAP, M.G University, Kottayam, Kerala for technical support. 


\section{References}

1. Norris D J. and. Bawendi M G, Phy Rev B, 1996, 53, 16338.

2. Ong H C and Chang R P H, Appl Phys Lett., 2001, 79(22), 3612-3614.

3. Aruldhas N, A Zaben, and A Gadanken, Chem Mater., 1999, 11(3), 806-813; DOI:10.1021/cm980670s

4. Ye Changhui, Fang Xiaosheng, Li Guanghai and Zhang Lide, Appl Phys Lett., 2004, 85(15), 3035.

5. Ma C, Moore D and Li J, Adv Mater., 2003, 15(3), 228-231; DOI:10.1002/adma.200390052

6. Li Q.and.Wang, C R, Appl Phys Lett.., 2003, 83, 359; DOI:10.1063/1.1591999

7. Mandal T, Meiti P K and Dasgupta C, Phys Rev B, 2012, 86, 024101.

8. $\quad$ Sridevi P, J Nano Sci Nano Tech., 2014, 2(1), 34-37.

9. Yanh H, Gao M, Wang Y X, Lili Y, Yongjun Z, Jihui L, Dandan W, Huilian L and Hougang F, Appl Surface Sci., 2008, 255(5), 2646-2650;

DOI:10.1016/j.apsusc.2008.08.001

10. Gilbert B, Zhang H, Chan B, Kunz M, Huang F and Banfield J F, Phy Rev B, 2006, 74, 115405

11. Chen H X, Shi D N, Qi J S and Baolin W, Physica E: Low-dimensional Systems Nanostructures, 2009, 42(1), 32-37.

12. Cheng B C, Xiao Y H and Wu G S and Zhang L D, Adv Funct Mater., 2014, 14, 913 919; DOI: 10.1002/adfm.200305097

13. Peng W Q, Cong, G W Qu, S C and Wang Z G, Nanotechnology, 2005, 16, 1469.

14. Nanda K K, Kruis F E and Fissan H, Phys Rev Lett., 2002, 89, 256103.

15. Abdul Khader M and Binny Thomas, Nanostructured Materials, 1998, 10(4), 593 600; DOI:10.1016/S0965-9773(98)00100-7

16. Nakamota Kazuo V, (Ed)., Infrared and Raman spectra of Inorganic and Coordination compounds, 1972.

17. Sibi Kurian, Shajo Sebastian, Jose Mathew and George K C, Indian J Pure Appl Phys., 2004, 42, 926.

18. Hou T H, Mao J, Zhu X D and Tu M J, Rare Met.., 2006, 25, 331-336.

19. Lappen P E and Lannoo M, Phys Rev B, 1989, 39(15), 10935;

DOI:10.1103/PhysRevB.39.10935

20. Tingting Ren, Holly R and Kristin M |P, Thin Solid Films, 2007, 515, 7976-7983.

21. Laura B L and Christoffer O K, Chem Mater., 1997, 9(6), 1302-1317;

DOI:10.1021/cm960441a 